\title{
A NOTE ON COUNTABLY GENERATED IDEALS IN RINGS OF CONTINUOUS FUNCTIONS ${ }^{1}$
}

\author{
CARL W. KOHLS
}

In a recent paper [1], Gillman introduced a number of clever devices to prove some results about countably generated ideals in $C(X)$. We show in this note how to modify the use of two of these devices so as to obtain several results of greater generality. The theorems presented here confirm that the class of countably generated ideals in $C(X)$ is quite restricted. For example, it was shown in [1] that a prime ideal in $C(X)$ cannot be countably generated if it is a free ideal or a nonmaximal $z$-ideal, but that certain prime ideals are countably generated. In $\S 2$, we complete the description of the countably generated prime ideals by determining precisely which of the fixed nonmaximal prime ideals that are countable unions of upper ideals are countably generated; and it turns out that the point associated with the ideal must be of a very special type.

This note may be regarded as a sequel to [1]; the reader should refer to that paper and to [2] for general background. We shall follow the notation and terminology of those works. The boundary of a set $S$ will be denoted by "bdy $S$. "

1. Various fixed ideals. For each fixed ideal $I$ in $C(X)$, we set $L(I)=\bigcap_{p \in \phi(I)} O_{p}$; clearly $L(I)$ is a $z$-ideal in $C(X)$. Now, although $I$ contains $\bigcap_{p \in \theta(I)} O^{p}[4,3.1]$, it may not contain $L(I)$. For example, it does not if $I$ is the intersection of a fixed maximal ideal and a free maximal ideal in $C(\mathbf{R})$. The results in this section indicate that countable generation of a fixed ideal $I$ depends largely upon the topological nature of $\phi(I)$ and the relationship of $I$ to $L(I)$. With the right conditions on these objects, we can even intersect the given fixed ideal with any free ideal without affecting the outcome.

LEMma. Let $I$ be a fixed ideal of $C(X)$ satisfying the following conditions: (1) Each point of bdy $\phi(I)$ has a countable base of neighborhoods; (2) I contains a $z$-ideal $H$ that contains $L(I)$ properly. Let $J$ be any free ideal or all of $C(X)$. Then $I \cap J$ is not countably generated.

Proof. Since $H \neq \boldsymbol{L}(I)$, there exists some $Z \in \boldsymbol{Z}[H]-\boldsymbol{Z}[\boldsymbol{L}(I)]$. By the definitions of $L(I)$ and $H$, there is a point $p \in$ bdy $\phi(I) \cap$ bdy $Z$.

Received by the editors September 16, 1960.

1 The preparation of this paper was sponsored by the National Science Foundation, under grant NSF G12171. 
The hypothesis on bdy $\phi(I)$ implies the existence of a sequence $S \subset X-Z$ that converges to $p$.

Now suppose that, on the contrary, $I \cap J=\left(f_{1}, f_{2}, \cdots\right)$, where we take each $\left|f_{m}\right| \leqq 1[2,2 \mathrm{~A}]$. The function defined to be zero on $\operatorname{cl}_{\beta X} Z$ and $\sum_{m} 2^{-m}\left|f_{m}\right|^{1 / 2}$ on $S$ is continuous on the compact set $S \cup_{\beta X} Z$, and hence it has a continuous extension to $\beta X$. Let the restriction to $X$ be denoted by $h$. Then $Z(h) \supset Z$, so $h \in H \subset I$. Next, let $t \in C^{*}(X)$ be such that $t^{*}=1$ on a neighborhood $U$ of $p$ and $t^{*}=0$ on a neighborhood (in $\beta X$ ) of $\theta(J)$. Then $t \in J[1,5.1]$, so $g=t h \in I \cap J$; and $g=h$ on $U$. For each point $q \in X-\phi(I)$, there is an $s \in C^{*}(X)$ such that $s(q)=1$, while $s^{*}=0$ on a neighborhood (in $\beta X$ ) of $\theta(I) \cup \theta(J)$, whence (applying [1, 5.1] again) $s \in L(I) \cap J \subset I \cap J$. Thus $\sum_{m} 2^{-m}\left|f_{m}\right|^{1 / 2}$ vanishes only on $\phi(I)$, and hence at no point of $S$. Therefore $\boldsymbol{Z}(h)$ is also disjoint from $S$.

By assumption, we have $g=s_{1} f_{1}+\cdots+s_{n} f_{n}$ for some $n \in \mathrm{N}$ and $s_{1}, \cdots, s_{n} \in C(X)$. At least one $f_{k}$ must be different from zero at each point of $U \cap S(k \leqq n)$. We now follow the proof of $[1,5.2]$, replacing " $F$ " by our " $p$," reaching again the inequality

$$
h(x)<2^{-n}\left(\left|f_{1}(x)\right|^{1 / 2}+\cdots+\left|f_{n}(x)\right|^{1 / 2}\right)
$$

for $x \in W \cap S$. But $W \cap S$ is nonempty, so we have arrived at a contradiction.

REMARK. A simplification of the technique just employed shows that the hypothesis of compactness in $[1,5.2]$ may be omitted.

TheOREM 1. Let $I$ be a z-ideal in $C(X)$ containing $L(I)$, and suppose that $\phi(I)$ satisfies condition (1) of the lemma, and in addition is completely separated from every disjoint closed set. Then $I$ is couniably generated if and only if $I=L(I)$ and $\phi(I)$ has a countable base of neighborhoods.

Proof. By $[2,1.15]$, the hypothesis on complete separation implies that $\boldsymbol{Z}[L(I)]$ is a base for the neighborhoods of $\phi(I)$.

Necessity. By the lemma, $I=L(I)$. Thus, $\boldsymbol{Z}[L(I)]$ has a countable base, whence so does the set of neighborhoods of $\phi(I)$.

Sufficiency. There is a countable base $\left\{\boldsymbol{Z}\left(f_{m}\right)\right\} \subset \boldsymbol{Z}[\boldsymbol{L}(I)]$ for the neighborhoods of $\phi(I)$. It follows from [2,1D.1] that any element of $L(I)$ is a multiple of some $f_{m}$.

The condition in Theorem 1 that $\phi(I)$ is completely separated from every disjoint closed set holds, of course, if $X$ is normal or $\phi(I)$ is compact, while the condition on bdy $\phi(I)$ holds if $X$ is first countable. Thus, special cases with simple hypotheses that are not especially restrictive are readily obtained. Notice also that Theorem 1 
covers, for all $X$, the interesting special case in which $\phi(I)$ is a single point with a countable base of neighborhoods (cf. [2, 4I.7]).

In the following examples, $\phi(I)$ is not completely separated from every disjoint closed set.

EXAMPLE 1. An ideal of the form $L(I)$ can be countably generated even though the corresponding closed set $\phi(I)$ does not have a countable base of neighborhoods. Let $X$ be the space $\beta \mathbf{R}-(\beta \mathbf{Q}-\mathbf{Q})$ (cf. $[2,6 \mathrm{P}])$. It is easily seen that the ideal $I=L(I)=\{f \in C(X): X-Z(f)$ is a bounded subset of $\mathbf{R}\}$ is countably generated. Let $\left\{U_{n}\right\}$ be any sequence of neighborhoods of $\phi(I)$. Since each $U_{n}$ must contain all irrationals outside some bounded set, we may choose an increasing sequence of distinct positive integers $\left\{k_{n}\right\}$ such that $U_{n}$ contains all irrationals with absolute value exceeding $k_{n}$. For each $n$, select any rational $q_{n} \in U_{n}$ with $k_{n}<q_{n}<k_{n+1}$. Then $V=X-\left\{q_{1}, q_{2}, \cdots\right\}$ is a neighborhood of $\phi(I)$, but $V$ contains no $U_{n}$. Thus, $\phi(I)$ does not have a countable base of neighborhoods.

EXAmple 2. An ideal of the form $L(I)$ can fail to be countably generated even though the corresponding closed set $\phi(I)$ has a countable base of neighborhoods. Let $T$ denote the set of rational numbers in the subspace $D$ of the space $\Gamma[2,3 \mathrm{~K}]$. Standard category and cardinality arguments may be applied to show that $T$ is not a zero-set in $\Gamma$. It follows that the ideal $I=L(I)=\bigcap_{p \in T} O_{p}$ in $C(\Gamma)$ is not countably generated. But the set $\phi(I)=T$ clearly has a countable base of neighborhoods.

2. Fixed prime ideals. We shall now describe the prime ideals in $C(X)$ that are countably generated. Since only a small amount of extra effort is required to provide a description for the more general class of pseudoprime ideals $[3, \S 3]$, we first present a theorem for that class. From $[2,7.15]$, any pseudoprime ideal $I$ in $C(X)$ contains $O^{p}$ for a unique $p$, and $M^{p}$ is the unique maximal ideal containing $I$. (Although the statement is for prime ideals, the proof requires only that the ideal be pseudoprime.) Also, the question of countable generation has already been settled for maximal ideals [1, 5.4]. Hence there is no real limitation in considering only a fixed ideal $[1,4.5]$ that is nonmaximal and contains some $\boldsymbol{O}_{\boldsymbol{p}}$.

Theorem 2. A nonmaximal pseudoprime ideal $I$ in $C(X)$ that contains $O_{p}$ is countably generated if and only if the following three conditions are satisfied:

(1) I contains an element $f$ such that $\boldsymbol{Z}(f)=\{p\}$.

(2) In the class of prime $z$-ideals contained properly in $M_{p}$, there is exactly one maximal element $Q$. 
(3) The ideal $I / Q$ in the ring $C / Q$ (where $Q$ is the ideal described in (2)) has a countable cofinal subset.

Proof. Necessity. If (1) did not hold, then $Z[I]$, being countably generated, would induce on $X-\{p\}$ a free, prime $z$-filter that is countably generated. But this contradicts $[1,4.5]$.

Suppose now that (2) does not hold. By (1), already established, $I$ must contain one of the prime $z$-ideals $P$, maximal in the class of those that are contained properly in $\boldsymbol{M}_{\boldsymbol{p}}$. Since, by our assumption, there is at least one other such prime $z$-ideal, there exist zero-sets $Z, Z^{\prime}$ such that $Z \in Z[P], p$ is in the closure of $Z^{\prime}-\{p\}$, and $Z \cap Z^{\prime}$ $=\{p\}$. Let $g \in C^{*}(X-\{p\})$ be a function such that $g=0$ on $Z-\{p\}$, and $g=1$ on $Z^{\prime}-\{p\}$; and let $\bar{g}$ be any real-valued extension of $g$ to $X$ (in this case not continuous, of course). Then the function $h=\bar{g} \sum_{m} 2^{-m}\left|f_{m}\right|^{1 / 2}$, where each $\left|f_{m}\right| \leqq 1$ and $I=\left(f_{1}, f_{2}, \cdots\right)$, is in $C(X)$. Now $\boldsymbol{Z}(h) \supset Z$, so $h \in P \subset I$. Thus, for some $n \in \mathbb{N}$ and $s_{1}, \cdots, s_{n} \in C(X)$, we have $h=s_{1} f_{1}+\cdots+s_{n} f_{n}$. Evaluating this equation on $Z^{\prime}$, we obtain

$$
\sum_{m} 2^{-m}\left|f_{m}(x)\right|^{1 / 2}=s_{1}(x) f_{1}(x)+\cdots+s_{n}(x) f_{n}(x), \quad x \in Z^{\prime} .
$$

As in the proof of the lemma in $\$ 1$, we now follow the proof of $[1$, 5.2] and, remembering that $p$ is in the closure of $Z^{\prime}-\{p\}$, obtain the usual contradiction. Thus, (2) holds.

To prove (3), we recall that the natural homomorphism of $C$ onto $C / Q$ is order-preserving. Thus every element in $I / Q$ is bounded above by a finite sum of positive integral multiples of the absolute values of the images of the generators; and the collection of these sums is countable.

Sufficiency. In the terminology of $[5,2.2]$, condition (2) implies that $\phi^{+}(p)$ consists of a single point. It follows immediately that the mapping $\phi$ is actually a homeomorphism; in other words, $p$ is actually a point of $\beta(X-\{p\})$, whence $X-\{p\}$ is $C^{*}$-embedded in $X$.

We show now that the countable cofinal subset of $I / Q$ generates $I / Q$. In fact, we can show more: Given $a, b \in I / Q$ with $0 \leqq a<b, a$ is a multiple of $b$. To prove this, we pick pre-images $f, g \in C(X)$ of $a, b$, respectively, with $0 \leqq f \leqq g$ on $X[2,13.5]$. Since $b \neq 0, \boldsymbol{Z}(g)=\{p\}$. It follows that $(f / g) \mid(X-\{p\}) \in C^{*}(X-\{p\})$. The desired result now follows immediately from the fact that $(f / g) \mid(X-\{p\})$ has a continuous extension to $p$.

We conclude that $I=\left(Q, f_{1}, f_{2}, \cdots\right)$, for some countable subset $\left\{f_{m}\right\}$ of $I-Q$. Thus, we need only show that $Q$ is countably generated. Again we can show more: Every element of $Q$ is a multiple of $f_{1}$ (or 
any other $\left.f_{m}\right)$. For, let $h \in Q$ be given, and set $k=\left(h / f_{1}\right) \mid(X-\{p\})$. Since $\boldsymbol{Z}\left(f_{1}\right)=\{p\}$, we have $k \in C(X-\{p\})$. Now if $k$ were unbounded in every deleted neighborhood of $p$, then $W=\{x \in X-\{p\}:|k|(x) \geqq 1\}$ would be a zero-set in $X-\{p\}$ disjoint from $\boldsymbol{Z}(h)-\{p\}$, but meeting every set in $\boldsymbol{Z}\left[O_{p}\right]$. Thus, $W$ would not belong to the free $z$-ultrafilter on $X-\{p\}$ that converges to $p$, while the collection consisting of $W$ and $\left\{Z-\{p\}: Z \in Z\left[O_{p}\right]\right\}$ would generate a $z$-filter on $X-\{p\}$ converging to $p$, which is impossible. Thus, $k$ is bounded in some deleted neighborhood of $p$; so there is a bounded continuous function that coincides with $k$ in that deleted neighborhood, and hence has a continuous extension to $p$. From this it follows that $k$ itself has a continuous extension, and so $h$ is a multiple of $f_{1}$.

Corollary. A nonmaximal prime ideal $I$ in $C(X)$ that contains $O_{p}$ is countably generated if and only if it satisfies conditions (1) and (2) of Theorem 2 and

(3') I is the union of a countable number of upper ideals.

Proof. $\left(3^{\prime}\right)$ is equivalent to (3) for nonmaximal prime ideals (cf. $[2,14$ C.3 $])$.

From $[5,2.2]$ and $[2,6.7]$, we see that condition (2) in Theorem 2 is actually equivalent (for a nonisolated $G_{\delta}$-point $p$ ) to the assumption that $p$ is a point from $\beta(X-\{p\}),{ }^{2}$ and thus amounts to an algebraic method of stating this topological hypothesis. There are several other topological statements equivalent to it, among them the statement that $X-\{p\}$ is $C^{*}$-embedded in $X$; see $[2,6.7]$. Note that this class of points includes the points for which $O_{p}$ is prime (see, e.g., [2, $14.21 \mathrm{ff}$.$] ), because in that case the prime z$-ideals contained in $M_{p}$ form a chain. But it is easily seen that there are other points as well; for example, take $X$ to be $R^{+} \cup\{p\}$, where $p \in \beta \mathrm{N}-\mathbf{N}$. Note also that this type of point $p$ cannot have a sequence of distinct points converging to it, by $[5,3.3]$ (see also $[2,9 \mathrm{~N} .2]) .^{3}$

3. Free ideals. In this section we use one of the ideas in $[1,5.5]$ to describe a large class of free ideals that are not countably generated.

TheOREM 3. Let $X$ be a locally compact, $\sigma$-compact space, and let $I$ be a free z-ideal in $C(X)$ such that $Z[I]$ contains a countable discrete set $Z$ of nonisolated points. Then $I$ is not countably generated.

\footnotetext{
2 The point $\sigma$ in the space $\Sigma[2,4 \mathrm{M}]$ is the classical example.

${ }^{3}$ It is amusing to observe that a non- $G_{\delta}$-point of this type can have a sequence converging to it-consider the Tychonoff plank $[2,8.20]$.
} 
Proof. Assume the contrary, and let $I=\left(f_{1}, f_{2}, \cdots\right)$. The hypotheses on $X$ imply that in its one-point compactification $X \cup\{p\}, p$ is a $G_{\delta}$-point. It is easy to see that $Z=\left\{x_{m}\right\}$ converges to $p$. By $[5,3.3]$, there exists a discrete family $\left\{V_{m}\right\}$ of zero-set neighborhoods of the points of $Z$ such that $p$ is the only limit point of $U_{m} V_{m}$. Let $\left\{U_{m}\right\}$ be a sequence of neighborhoods of the points of $Z$ such that $\operatorname{cl}_{X} U_{m}$ $C$ int $V_{m}$, and $\operatorname{cl}_{X} U_{m}$ is compact. Since $x_{m}$ is not isolated, we may pick a $g_{m} \in C\left(\operatorname{cl}_{X} U_{m}\right)$ that belongs to $M_{x_{m}}$ but not to the ideal in $C\left(\operatorname{cl}_{X} U_{m}\right)$ generated by those functions $f_{k} \mid\left(\operatorname{cl}_{X} U_{m}\right)$ that vanish at $x_{m}$. We extend each $g_{m}$ to a function in $C^{*}\left(V_{m}\right)$.

Since $\operatorname{cl}_{X} U_{m}$ is completely separated from $X$-int $V_{m}$, there exists, for each $m \in \mathrm{N}$, a function in $C(X)$ that coincides with $g_{m}$ on $\mathrm{cl}_{X} U_{m}$ and vanishes outside of $V_{m}$. It is easy to see that the sum $g$ of these functions is in $C(X)$; and $g \mid\left(\operatorname{cl}_{X} U_{m}\right)=g_{m}$. Now $\boldsymbol{Z}(g) \supset Z$, so $g \in I$; and hence $g=s_{1} f_{1}+\cdots+s_{n} f_{n}$, for some $n \in \mathbf{N}$ and $s_{1}, \cdots, s_{n} \in C(X)$. Since $Z \cap\left(\bigcap_{k=1}^{n} \boldsymbol{Z}\left(f_{k}\right)\right) \in \boldsymbol{Z}[I]$, it is nonempty, and this yields a contradiction as in $[1,5.5]$.

Obviously not every ideal of the type described in the theorem satisfies the necessary condition for countable generation given in $[1,4.4]$, because every noncompact zero-set can be embedded in a free $z$-ultrafilter. On the other hand, there are many ideals that do satisfy this condition-for example, the ideal of all functions that vanish on all but a finite number of points of $Z$, i.e., the smallest $z$ ideal containing the ideal of functions with compact support and any function whose zero-set is $Z .^{4}$ (This is a direct generalization of the example given in $[1,5.5]$.)

\section{REFERENCES}

1. L. Gillman, Countably generated ideals in rings of continuous functions, Proc. Amer. Math. Soc. vol. 11 (1960) pp. 660-666.

2. L. Gillman and M. Jerison, Rings of continuous functions, Princeton, Van Nostrand, 1960.

3. L. Gillman and C. W. Kohls, Convex and pseudoprime ideals in rings of continuous functions, Math. Z. vol. 72 (1960) pp. 399-409.

4. C. W. Kohls, Ideals in rings of continuous functions, Fund. Math. vol. 45 (1957) pp. $28-50$.

5. - Prime ideals in rings of continuous functions. II, Duke Math. J. vol. 25 (1958) pp. $447-458$.

\section{UNIVERSITY OF ILLINOIS}

${ }^{4}$ Recall that every $\sigma$-compact space is realcompact $[2,8.2]$, and that $Z$ is $C^{*}$ embedded in $X[5,3.3]$. 